[24] Yao JQ. Introduction of THz-wave and its applications. J Chongqing Univ Posts Telecommun (Nat Sci). 2010;22:703.

[25] Jin YS, Kim GJ, Jeon SG. Terahertz dielectric properties of polymers. J Korean Phys Soc. 2006;49:513.

[26] Fischer BM, Wietzke S, Reuter M, Peters O, Gente R. Investigating material characteristics and morphology of polymers using terahertz technologies. IEEE Trans Terahertz Sci Technol. 2013;3:259.

[27] Fedulova E, Nazarov MM, Angeluts A, Kitai MS, Sokolov VI, Shkurinov AP. "Studying of dielectric properties of polymers in the terahertz frequency range", Proceedings of SPIE, 8337, 83370I; 2012.

[28] Madbouly SA, Mansour AA, Abdou NY. Molecular dynamics of amorphous/crystalline polymer blends studied by broadband dielectric spectroscopy. Eur. Polym. J. 2007;43:1892.

[29] Yomogida Y, Sato Y, Yamakawa K, et al. Comparative dielectric study of pentanol isomers with terahertz time-domain spectroscopy. J. Mol. Struct. 2010;970:171.

[30] Chen K, Schweizer KS. Theory of physical aging in polymer glasses. Phys Rev E. 2008;78:031802.

[31] Bandyopadhyay A, Odegard GM. Molecular modeling of physical aging in epoxy polymers. J Appl Polym Sci. 2013;128: 660 .

[32] Kremer F, Serghei A, Sangoro JR, Tress M. Broadband Dielectric Spectroscopy, New York; 2003.

[33] Peiponen KE, Zeitler A, Kuwata-Gonokami M. Terahertz Spectroscopy and Imaging. Berlin: Springer; 2013.

[34] Chen XL, Xin C, Zhu ZY. THz-TDS spectra study of polymer materials with different polarity. J Infrared Millimeter Waves. 2013;32:150.

[35] Zhao KS. Dielectric Spectroscopy and Application. Beijing: Chemical Industry Press; 2008.

[36] Sasabe H, Saito S. Effects of temperature and pressure on the dielectric constant in non-polar polymers. Polym J. 1972;3:749.

[37] Valiskó M, Boda D. Dielectric constant of the polarizable dipolar hard sphere fluid studied by Monte Carlo simulation and theories. Condens. Matter Phys. 2005;8:357.

[38] Schwartz GA, Cerveny S, Marzocca ÁJ, Gerspacher M, Nikiel L. Thermal aging of carbon black filled rubber compounds. I. Experimental evidence for bridging flocculation. Polymer. 2003; 44:7229.

[39] Cunneen JI. Oxidative aging of natural rubber. Rubber Chem. Technol. 1968;182.

[40] Choi S. Influence of rubber composition on change of crosslink density of rubber vulcanizates with EV cure system by thermal aging. J Appl Polym Sci. 2000;75:1378.

[41] Guo L, Huang G, Zheng J, Li GX. Thermal oxidative degradation of styrene-butadiene rubber (SBR) studied by 2D correlation analysis and kinetic analysis. J Therm Anal Calorim. 2014;115: 647.

[42] Hamed GR, Zhao J. Tensile behavior after oxidative aging of gum and black-filled vulcanizates of SBR and NR. Rubber Chem Technol. 1999;72:721.

[43] Chinese Materials Engineering Canon: Polymer Materials Engineering. Beijing: China Chemistry Industry Press; 2006.
[44] Xu JZ, Zhang XC. Terahertz Science Technology and Applications. Beijing: Peking University Press; 2007.

[45] Institute of synthetic materials aging, Ministry of chemical industry, Polymer Materials Aging and Resistance to Aging. Beijing: Chemical Industry Publishing House; 1979.

[46] Bélan F, Bellenger V, Mortaigne B, Verdu J. Relationship between the structure and hydrolysis rate of unsaturated polyester prepolymers. Polym Degrad Stab. 1997;56:301.

[47] Johari GP. Structural changes and molecule mobility during physical ageing of glasses. J Phys Coll. 1985;46:C8-567.

How to cite this article: Chang T, Zhang X, Cui H-L. Evolution of terahertz dielectric permittivity of rubber during thermo-oxidative aging. Microw Opt Technol Lett. 2017;60:127-134. https://doi.org/10.1002/mop. 30930

Received: 13 June 2017

DOI: $10.1002 /$ mop.30929

\section{A novel circularly polarized filtering antenna with high out- of-band radiation rejection level}

\section{Meijun $\mathbf{Q u}^{\mathbf{1}} \quad$ | Mingxing $\mathbf{L i}^{\mathbf{2}}$ | \\ Lidan $\mathbf{Y a o}^{2}$ | Menlou $\mathrm{Rao}^{3}$ | \\ Shufang Li ${ }^{1}$ [1) | Li Deng ${ }^{1}$}

${ }^{1}$ School of Information and Communication Engineering, Beijing Key Laboratory of Network System Architecture and Convergence, Beijing University of Posts and Telecommunications, Beijing 100876, China

${ }^{2}$ School of Electronic Engineering, Beijing Key Laboratory of Work Safety Intelligent Monitoring, Beijing University of Posts and

Telecommunications, Beijing 100876, China

${ }^{3}$ Department of Electrical Engineering and Computer Science, University of Michigan, Ann Arbor, Michigan 48109

\section{Correspondence}

Shufang Li, School of Information and Communication Engineering, Beijing Key Laboratory of Network System Architecture and Convergence, Beijing University of Posts and Telecommunications, P.O. Box. 171, Beijing 100876, China.

Email: bupt_paper@126.com

\section{Abstract}

In this paper, a circularly polarized filtering antenna with high out-of-band radiation rejection level is proposed. The entire design consists of a patch and a filtering feeding network, which are connected by metallic pins. First, a highselectivity filtering power divider is constructed utilizing 
two shunted bandpass filter. Next, a $90^{\circ}$ phase difference could be realized by adjusting the length of the two outputs of the power divider. The measured transmission coefficient of the feeding network is $6.3 \mathrm{~dB}$ at $2 \mathrm{GHz}$. The measured axial ratio is below $3 \mathrm{~dB}$ within the operating band of the proposed antenna, which represents the effectiveness of the proposed feeding network. In addition, the obvious radiation nulls can be found from the antenna measurement because of the transmission zeros introducing by the filtering power divider. The measured gain is $5.73 \mathrm{~dB}$ at $2 \mathrm{GHz}$ and decreases dramatically in stopband. Specially, the measured gain is -30 and $-41 \mathrm{~dB}$ at 1.95 and $2.06 \mathrm{GHz}$. The proposed antenna has an excellent filtering characteristic compared with those without the filtering feeding network. To sum up, the proposed antenna and filtering power divider are promising in the modern wireless communication systems.

\section{KE Y W ORDS}

bandpass filter, circularly polarized antenna, feeding network, filtering response, radiation null

\section{1 | INTRODUCTION}

Filters and antennas are two indispensable components in RF front-end. Traditionally, they are designed separately and then are cascaded into the system. The input ports are assumed to be ideal $50 \Omega$ interface in the designing of filters and antennas individually, which are not accurate in practical and leading a deteriorated performance. Recently, the integration of bandpass filter and antenna into a subsystem has drawn increasing attentions. The match circuit is greatly simplified compared with the traditional cascaded design while the insertion loss caused by filter is decreased. The common method to obtain the filtering antenna is using the antenna radiator as the laststage resonator of the filter. ${ }^{1-3}$ Whereas, implementing multiple resonators increases the size of the design and the gain of this kind of filtering antenna is relatively low.

There are many approaches ${ }^{4-12}$ reported to realize filtering antennas, featuring filter-like frequency response both for return loss and antenna gain, nowadays. In Ref. [4], a second order bandpass filter with microstrip patch antenna is coupled by a rectangular aperture. Thus, a sharp transit is achieved from out-band to in-band at both sides of the operating frequency band. The multistub microstrip line is adopted to excite two separate U-shaped patches at different frequencies. $^{5}$ The multistub feed line can generate two controllable resonant modes as well as two nulls in realized gain at boresight direction. In Ref. [6], modified metasurface is introduced to generate a radiation null near the upper band edge. A shorting via is embedded in the feeding circuit to provide another radiation null at the lower band edge. However, the aforementioned references are about the designing of the linearly polarized antenna. In Ref. [7], a nearly square patch radiator with truncated corners is excited by a bandpass filter circuit through a metallic pin. The feeding position of the pin on the patch is offset from the center in order to obtain low circularly polarized axial ratio. ${ }^{8}$ To obtain a filtering circularly polarized antenna (FCPA), a filtering quadrature coupler is adopted to excite a hollow dielectric resonator antenna.

In this paper, a right-handed circularly polarized antenna implanting the filtering response is proposed. A bandpass filter and power divider are designed in section 2 . The proposed filtering power divider offers several advantages, including high selectivity, low insertion loss, wide stopband suppression, and excellent in-band isolation. The FCPA is presented in section 3. By adjusting the lengths of the two outputs of the proposed filtering power divider, $90^{\circ}$ phase difference of the feeding network is realized. Therefore, the circularly polarized wave is excited by feeding network to the patch antenna. Due to the transmission zeros of the filtering power divider, the obvious radiation nulls can be found. Hence, a high out-of-band radiation rejection level of the proposed antenna is achieved. From the measured results, a gain of $5.35 \mathrm{~dB}$ at $2 \mathrm{GHz}$ is obtained while the gain decreases dramatically in stopband.

\section{2 | BANDPASS FILTER AND FILTERING POWER DIVIDER DESIGN}

\section{1 | Bandpass filter loaded with open stubs based on dual-band resonator}

The circuit structure and layout of the proposed bandpass filter $(\mathrm{BPF})$ are illustrated in Figure 1A-B. The proposed BPF is based on a quarter-wavelength side-coupled ring resonator $\left(Z_{\mathrm{e} 1}, Z_{\mathrm{o} 1}, \theta\right.$ and $\left.Z_{\mathrm{e} 2}, Z_{\mathrm{o} 2}, \theta\right)$, which can be seen from Figure 1A. The input/output Ports 1,2 are connected the ring resonator by a coupled line $\left(Z_{\mathrm{e} 3}, Z_{\mathrm{o} 3}, \theta\right)$ which links stepped impedance transform elements $\left(Z_{1}, Z_{2}\right)$. Two stepped open stubs $\left(Z_{3}, Z_{4}\right)$ are shunted connected in the input/output Ports 1,2 , respectively. The characteristic impedances of the two microstrip lines at input/output ports are all $Z_{0}=50 \Omega$ and all the electric length $\theta$ is $90^{\circ}$. The introduction of the stepped impedance transform elements and open stubs is used to realize four additional transmission zeros near the passband. The four out-of-band transmission zeros can be adjusted by varying the impedances $\left(Z_{1}, Z_{2}, Z_{3}, Z_{4}\right)$. A conventional uniform ring resonator is dual-mode ${ }^{13,14}$ and can be adopted as the basic cell resonator for higher order bandpass filter applications therefore. 


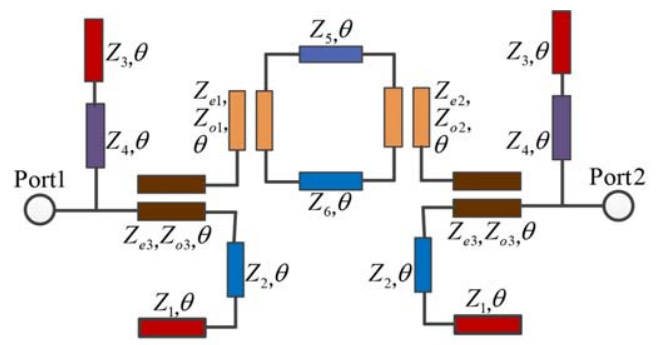

(A)

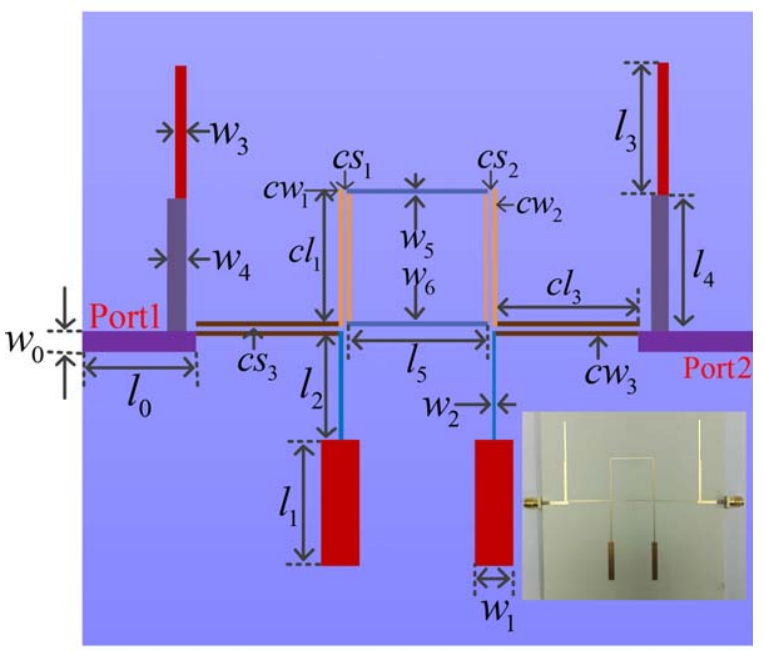

(B)

FIGURE 1 The circuit structure A, and layout B, of the proposed bandpass filter with physical-size definition. [Color figure can be viewed at wileyonlinelibrary.com]

A prototype of the BPF is fabricated on substrate of RO4350B with relative permittivity constant of 3.48 , thickness of 30 mil and loss tangent of 0.0037. Figure 2A and B indicates the scattering parameters of the proposed BPF. It can be seen from the Figure 2A that the measured reflection coefficient below $-10 \mathrm{~dB}$ over a bandwidth of $18.9 \%$ from
1.77 to $2.14 \mathrm{GHz}$ while the insertion loss is close to $2 \mathrm{~dB}$ due to the error of fabrication and the connector. Obvious transmission zeros can be found in Figure 2A, and the reflection coefficient below $-20 \mathrm{~dB}$ is greater than the band which is from 0 to $1.67 \mathrm{GHz}$ and 2.26 to $5.5 \mathrm{GHz}$, thus wide outof-band suppression and high selectivity of the proposed $\mathrm{BPF}$ can be realized. The measured group delay of the proposed BPF from Figure 2B is below 5 ns within the frequency band from 1.8 to $2.2 \mathrm{GHz}$, and is $3.34 \mathrm{~ns}$ at $2 \mathrm{GHz}$. There is an excellent agreement between the simulated and measured results. The final optimized geometrical parameters of the proposed BPF are tabulated in Table 1.

\subsection{Design of the filtering power divider based on the proposed BPF}

Two BPFs can be integrated into a power divider to achieve improved frequency selectivity ${ }^{15,16}$ and decrease the interference and noise hence. The proposed filtering power divider (FPD) shunts two aforementioned BPFs. Note that the characteristic impedances of the input (Port 1) and two outputs (Ports $2,3)$ are $50 \Omega$. The port impedance of the BPF should be viewed as $100 \Omega$ because of the shunting connection. The circuit scheme and layout of the FPD are plotted in Figure 3A-B while the design parameters are listed in Table 2. An isolation resistor is add to improve the isolation of the two outputs of the FPD $(r=600 \Omega)$.

To validate the effectiveness of the proposed FPD, a prototype is also manufactured on substrate of RO4350B. Figure 4 displays the measured and simulated scattering parameters of the proposed FPD. The measured results show that the proposed FPD is operating from 1.95 to $2.06 \mathrm{GHz}$. The measured transmission coefficient is $6.3 \mathrm{~dB}$ at $2 \mathrm{GHz}$, which represents that the proposed FPD has a low insertion loss relatively. Nevertheless, the insertion loss is increased

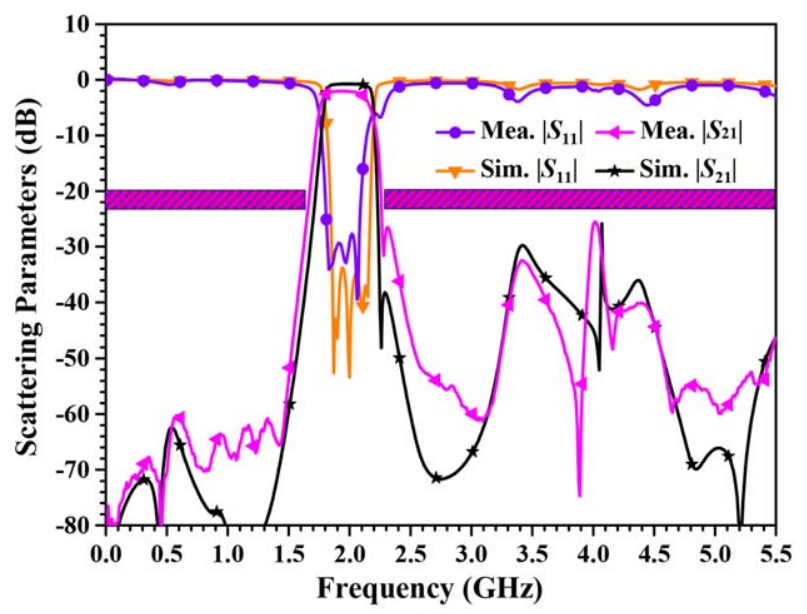

(A)

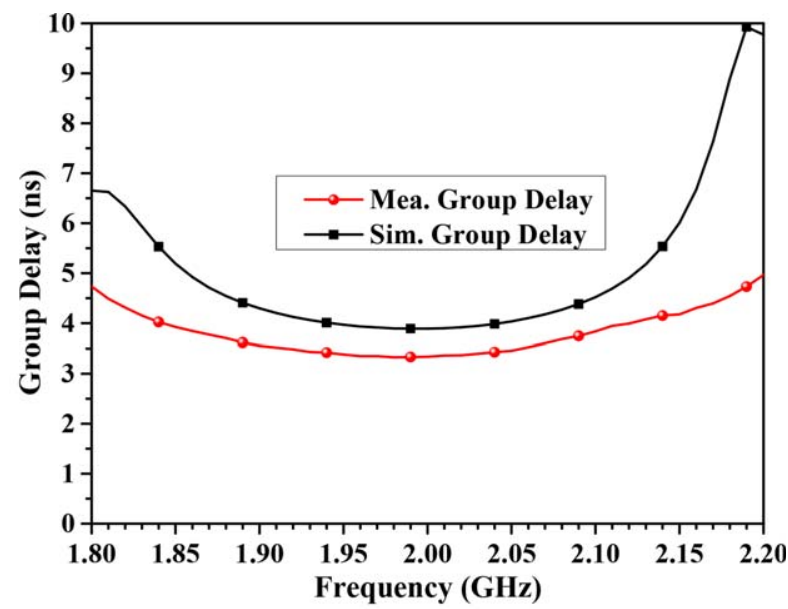

(B)

F I G URE 2 The measured and simulated results of proposed BPF: A scattering parameters, B group delay. [Color figure can be viewed at wileyonlinelibrary.com] 
TABLE 1 The geometrical parameters of the proposed BPF (Unit: mm)

$\begin{array}{llllllll}\text { Parameters } & \text { Values } & \text { Parameters } & \text { Values } & \text { Parameters } & \text { Values } & \text { Parameters } & \text { Values } \\ w_{0} & 1.66 & l_{0} & 15 & w_{1} & 2.83 & l_{1} & 21.64 \\ w_{2} & 0.27 & l_{2} & 23.4 & w_{3} & 0.915 & l_{3} & 22.67 \\ w_{4} & 1.42 & l_{4} & 22.3 & w_{5} & 0.36 & l_{5} & c 2.42 \\ w_{6} & 0.25 & c w_{1} & 0.17 & c s_{1} & 0.48 & 0.14 & c l_{3} \\ c w_{2} & 0.17 & c s_{2} & 0.48 & c w_{3} & / & / & \end{array}$

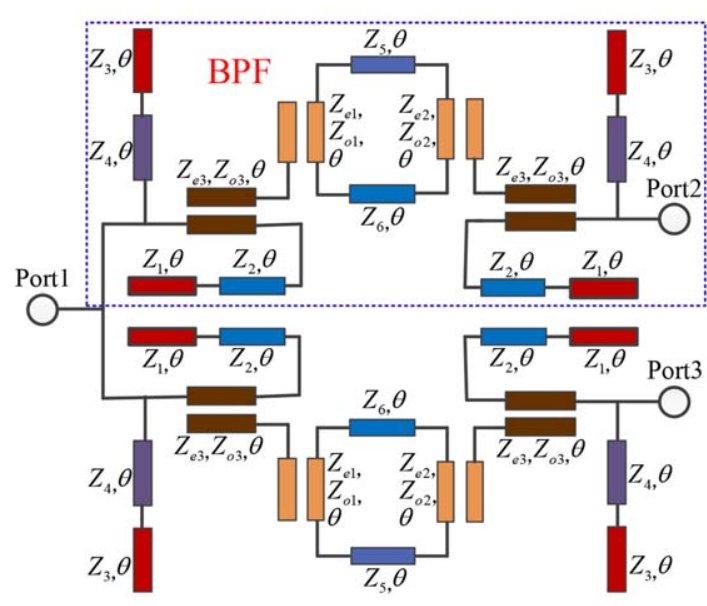

(A)

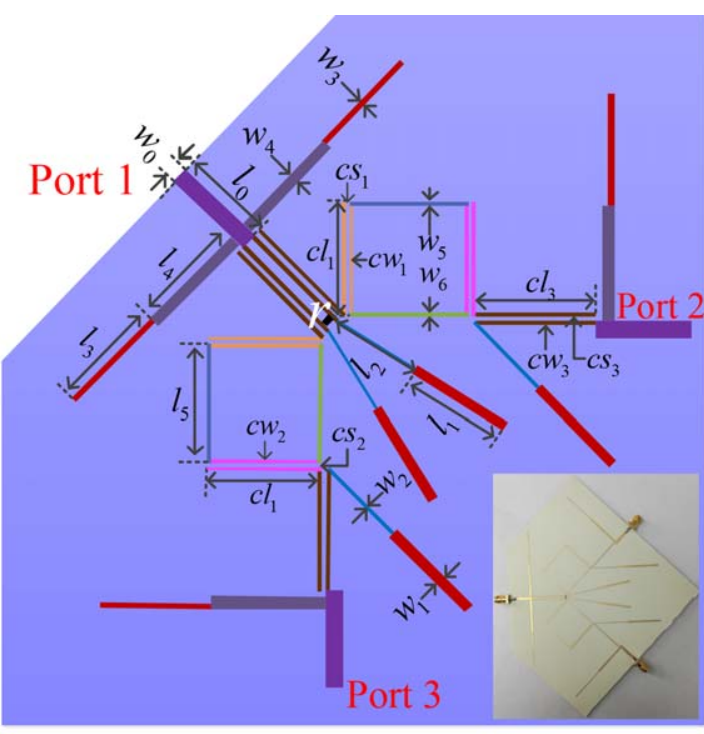

(B)

F IG URE 3 The circuit structure A, and layout B, of the proposed filtering power divider with physical-size definition. [Color figure can be viewed at wileyonlinelibrary.com]

dramatically out of the working band and sharp skirt selectivity is achieved. The measured stopband below $-20 \mathrm{~dB}$ is greater than the band which is from 1 to $1.79 \mathrm{GHz}$ and 2.09 to $3 \mathrm{GHz}$. The $\left|S_{21}\right|$ is $-59 \mathrm{~dB}$ at $1.45 \mathrm{GHz}$ and $-62 \mathrm{~dB}$ at $2.56 \mathrm{GHz}$ for instance. To enhance the isolation between two outputs, an isolation resistor $(r)$ is added in proposed FPD.

TABLE 2 The geometrical parameters of the proposed FPD and FCPA (Unit: mm)

\begin{tabular}{|c|c|c|c|c|c|c|c|}
\hline Parameters & Values & Parameters & Values & Parameters & Values & Parameters & Values \\
\hline$w_{0}$ & 1.66 & $l_{0}$ & 15 & $w_{1}$ & 0.915 & $l_{1}$ & 22.65 \\
\hline$w_{2}$ & 0.24 & $l_{2}$ & 22.65 & $w_{3}$ & 0.3 & $l_{3}$ & 23.36 \\
\hline$w_{4}$ & 1.05 & $l_{4}$ & 22.55 & $w_{5}$ & 0.37 & $l_{5}$ & 24 \\
\hline$w_{6}$ & 0.24 & $c w_{1}$ & 0.26 & $c s_{1}$ & 0.9 & $c l_{1}$ & 23.44 \\
\hline$c w_{2}$ & 0.214 & $c s_{2}$ & 0.72 & $c w_{3}$ & 0.15 & $\mathrm{cl}_{3}$ & 23.76 \\
\hline$c s_{3}$ & 0.42 & $l_{6}$ & 10.25 & $l_{7}$ & 54.23 & $l_{8}$ & 10.25 \\
\hline$l_{9}$ & 14.17 & $l_{10}$ & 53.36 & $l$ & 60.8 & $l_{\mathrm{a}}$ & 130 \\
\hline$l_{\mathrm{b}}$ & 95 & $l_{\mathrm{c}}$ & 17.6 & $h$ & 5 & $d$ & 18 \\
\hline
\end{tabular}




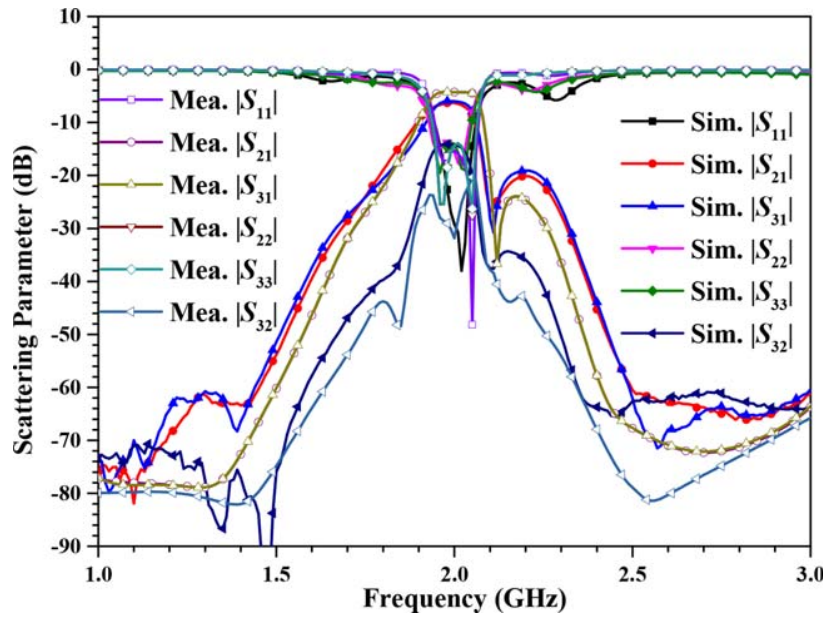

F IGURE 4 The measured and simulated scattering parameters of proposed FPD. [Color figure can be viewed at wileyonlinelibrary.com]

The measured in-band isolation is better than $14 \mathrm{~dB}$ ranged from 1.95 to $2.06 \mathrm{GHz}$, a relatively high isolation is obtained hence. Excellent agreement between the simulated and measured results is acquired. In addition, it can be expected that this power divider will be widely applied in the modern wireless communication systems.

\section{3 | FCPA DESIGN}

Figure 5A displays the 3-D geometry of the proposed FCPA. It consists of the feeding network based on the proposed FPD in the bottom layer and a rectangular patch in the top layer. As can be seen from Figure 5C, the feeding circuit excites the patch antenna with two metallic pins when the feeding position is properly adjusted. To achieve the circularly polarized wave, the lengths of the two outputs of the FPD are modified which can be found from Figure 5B. The length of the Port 2 is longer than that of the Port 3, a quarter wavelength approximately, a $90^{\circ}$ phase difference is realized between the two outputs of the feeding network. Note that the values of the parameters of feeding network are the same with those of the FPD, except the added variables $l_{6}, l_{7}, l_{8}, l_{9}$, and $l_{10}$. The final optimized geometrical parameters are listed in Table 2.

The proposed FCPA is fabricated and measured for demonstration. The substrates of the patch antenna and feeding circuit are both RO4350B. As seen from Figure 6, the 10-dB impedance bandwidth is from 1.96 to $2.07 \mathrm{GHz}$, agreeing reasonably with the simulated value $(1.94-2.06 \mathrm{GHz})$. Thanks to the transmission zeros of the FPD, the obvious radiation nulls can be found in Figure 6A. Hence, high outof-band radiation rejection level is achieved. A measured gain of $5.34 \mathrm{~dB}$ at $2 \mathrm{GHz}$ is obtained a little bit lower than the simulated value $(7.36 \mathrm{~dB})$. The gain decreases significantly in stopband. Specially, the measured gain is -30 and $-41 \mathrm{~dB}$ at 1.95 and $2.06 \mathrm{GHz}$. There is an excellent filtering feature compared with antennas without the filtering feeding network. The measured axial ratio is below $3 \mathrm{~dB}$ within the operating band from the Figure 6B. It also proves that the pure circularly polarized wave feeds the patch antenna by the proposed feeding circuit. The Figure 6C,D plot the 2-D radiation pattern of the proposed FCPA in $\mathrm{XOZ}$ and $Y O Z$ plane at $2 \mathrm{GHz}$. The gain of right-handed circularly polarization is much stronger than that of the left-handed circularly polarization. Thus, the proposed antenna is a filtering right-handed circularly polarized antenna. Changing the length of the two

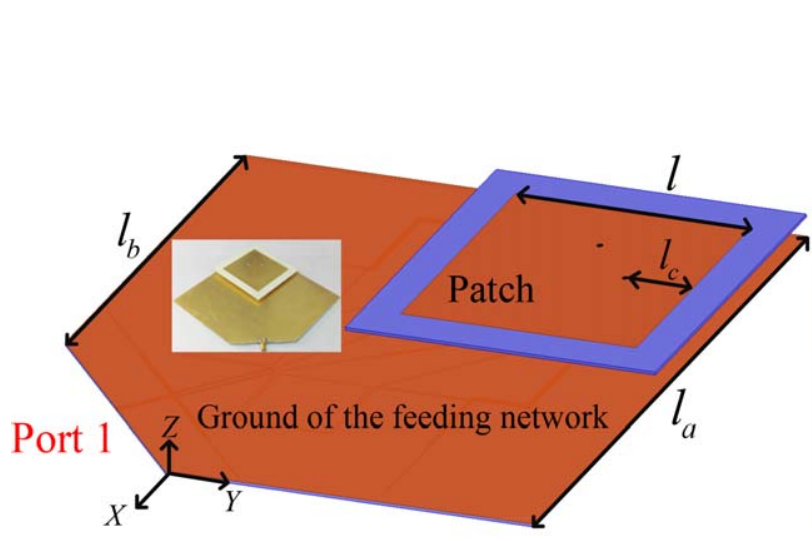

(A)

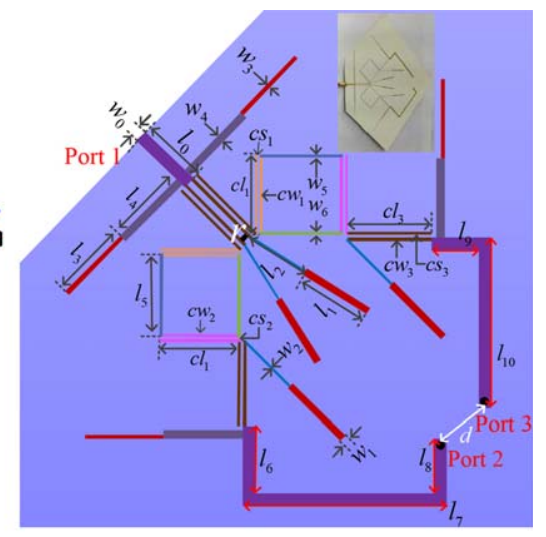

(B)

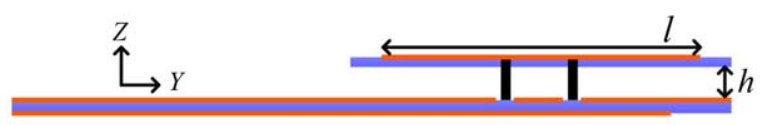

(C)

FIG URE 5 Schematic of the proposed FCPA: A, 3-D view of the proposed FCPA, B, layout of the feeding circuit in the proposed FCPA, and C, lateral view of the proposed FCPA. [Color figure can be viewed at wileyonlinelibrary.com] 


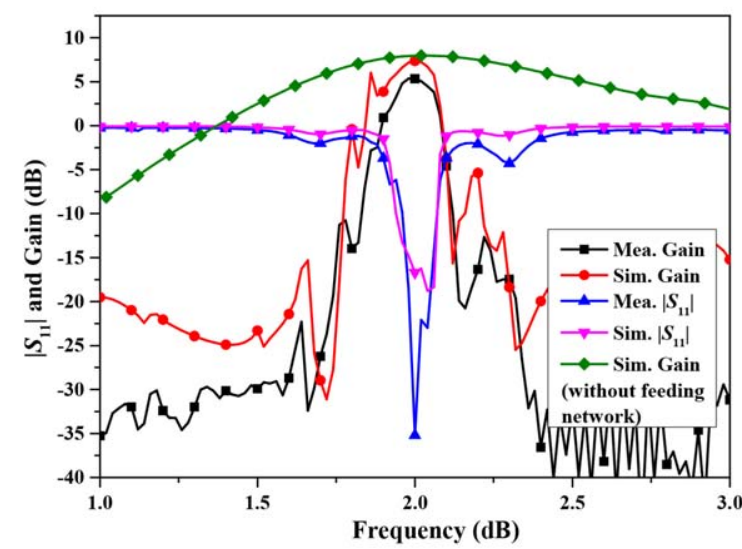

(A)

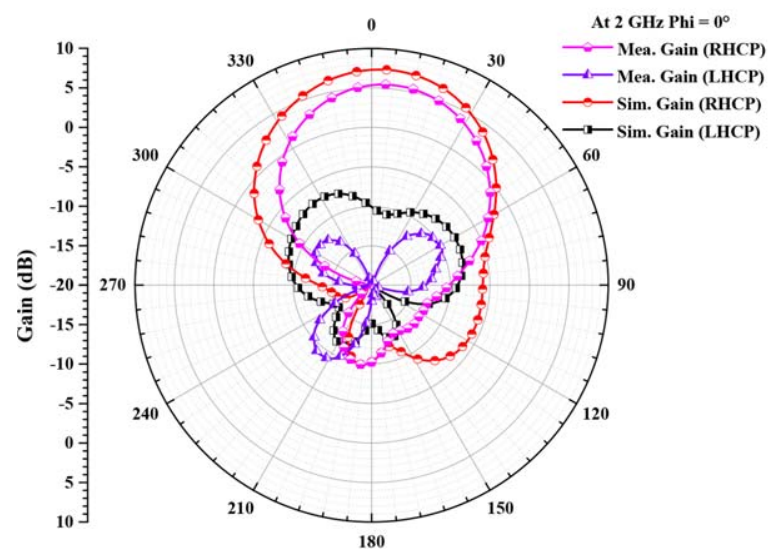

(C)

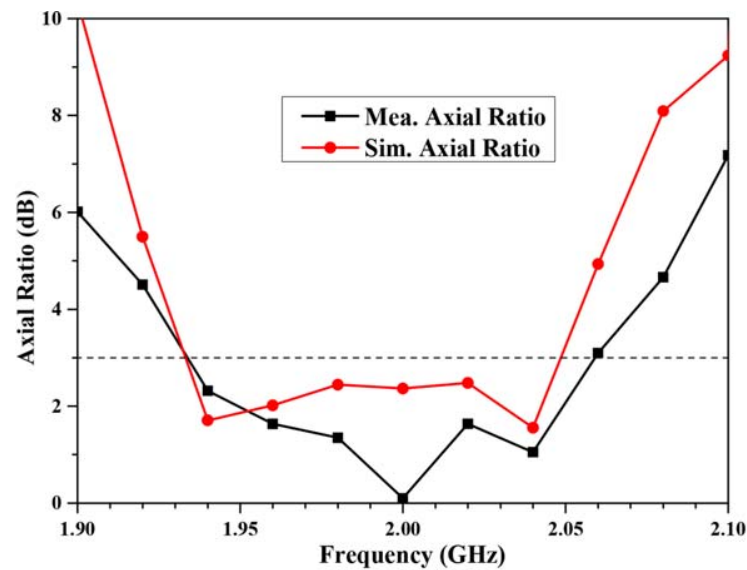

(B)

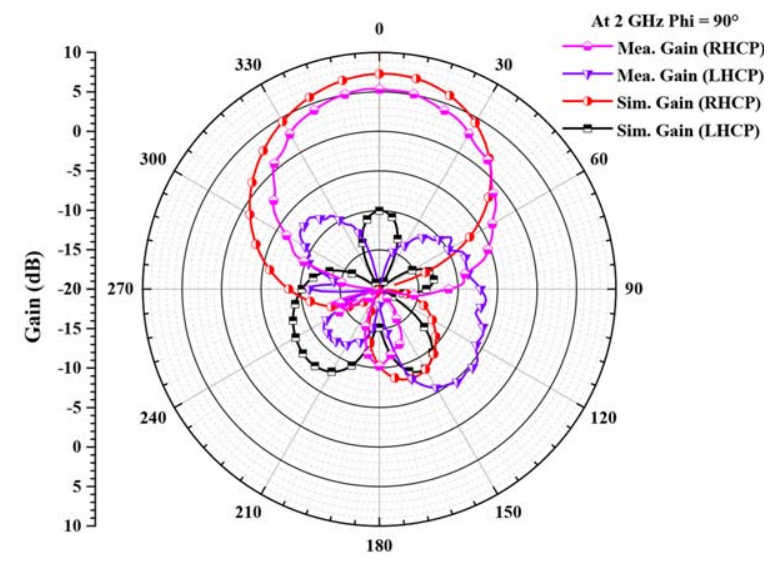

(D)

FIG URE 6 The measured and simulated scattering parameters and gain A, of the proposed FCPA, B, axial ratio, C-D, radiation pattern of the proposed FCPA in $\mathrm{XOZ}$ and $\mathrm{YOZ}$ planes at $2 \mathrm{GHz}$. [Color figure can be viewed at wileyonlinelibrary.com]

outputs of the feeding network, a left-handed circularly polarized antenna can also be obtained. It should be mentioned that the scattering parameters of the BPF, FPD, and FCPA are measured by Vector Network Analyzer (Rohde \& Schwarz ZVA-8), the gain of the FCPA is obtained in SATIMO measurement system in a microwave anechoic chamber.

\section{4 | CONCLUSIONS}

In this paper, a right-handed circularly polarized antenna with the filtering feeding network is presented. To excite the circularly polarized wave, a filtering power divider based on a bandpass filter is designed first. By adjusting the lengths of the two outputs of the proposed filtering power divider, $90^{\circ}$ phase difference is realized. The proposed filtering power divider offers several advantages, including high selectivity, low insertion loss, wide stopband suppression and excellent in-band isolation. Then, the feeding network is connected to the patch antenna by metallic pins. From the measured results, high out-of-band radiation rejection level is achieved.
A measured gain of $5.34 \mathrm{~dB}$ at $2 \mathrm{GHz}$ is obtained while the gain decreases dramatically in stopband. Specially, the gain is -30 and $-41 \mathrm{~dB}$ at 1.95 and $2.06 \mathrm{GHz}$. There is an excellent filtering feature compared with antennas without the filtering feeding network. Therefore, this proposed FCPA is suitable in modern wireless communication systems to prevent the interference and noise.

\section{ACKNOWLEDGMENTS}

This work was supported by National Natural Science Foundations of China (Nos. 61427801 and 61601040).

\section{ORCID}

Shufang Li (iD http://orcid.org/0000-0002-9359-8202

\section{REFERENCES}

[1] Chen X, Zhao F, Yan L, Zhang W. A compact filtering antenna with flat gain response within the passband. IEEE Antennas Wirel Propag Lett. 2013;12:857-860. 
[2] Lin C, Chung S. A compact filtering microstrip antenna with quasi-elliptic broadside antenna gain response. IEEE Antennas Wirel Propag Lett. 2011;10:381-384.

[3] Chuang C, Chung S. A compact printed filtering antenna using a ground-intruded coupled line resonator. IEEE Trans Antennas Propag. 2011;59(10):3630-3637.

[4] Mao C, Gao S, Wang Z, et al. Integrated filtering-antenna with controllable frequency bandwidth. In: 2015 9th European Conference on Antennas and Propagation; 2015:1-5.

[5] Zhang X, Zhang Y, Pan Y, Duan W, Low-profile dual-band filtering patch antenna and its application to LTE MIMO system, IEEE Trans Antennas Propag, 2017;65(1):103-113.

[6] Pan Y, Hu P, Zhang X, Zheng S. A low-profile high-gain and wideband filtering antenna with metasurface. IEEE Trans Antennas Propag. 2016;64(5):2010-2016.

[7] JiangGregory ZM, Werner D. Design and experimental investigation of a compact circularly polarized integrated filtering antenna for wearable biotelemetric devices. IEEE Trans Biomed Circ Syst. 2016;10(2):328-338.

[8] Xiang B, Zheng S, Pan Y, Li Y. Wideband circularly polarized dielectric resonator antenna with bandpass filtering and wide harmonics suppression response. IEEE Trans Antennas Propag. 2017;65(4):2096-2101.

[9] $\mathrm{Hu} \mathrm{H}$, Chen F, Chu Q. Novel broadband filtering slotline antennas excited by multimode resonators. IEEE Antennas Wirel Propag Lett. 2017;16:489-492.

[10] Hu P, Pan Y, Zhang X, Zheng S. A compact filtering dielectric resonator antenna with wide bandwidth and high gain. IEEE Trans Antennas Propag. 2016;64(8):3645-3651.

[11] Liu W, Chen Z, Qing X. Metamaterial-based low-profile broadband aperture-coupled grid-slotted patch antenna. IEEE Trans Antennas Propag. 2015;63(7):3325-3329.

[12] Sun G, Wong S, Zhu L, Chu Q. A compact printed filtering antenna with good suppression of upper harmonic band. IEEE Antennas Wirel Propag Lett. 2016;15:1349-1352.

[13] Salleh M, Prigent G, Pigaglio O, Crampagne R. Quarter-wavelength side-coupled ring resonator for bandpass filters. IEEE Trans Microwave Theory Tech. 2008;56(1):156-162.

[14] Wu Y, Hu B, Nan L, Liu Y. Compact high-selectivity bandpass filter using a novel uniform coupled-line dual-mode resonator. Microwave Opt Technol Lett. 2015;57(10):2355-2358.

[15] Jiang Z, Werner D. A co-designed wideband circularly polarized integrated filtering antenna. In: 2015 Asia-Pacific Microwave Conference 2015;3:1-3.

[16] Li Y, Xue Q, Zhang X. Single- and dual-band power dividers integrated with bandpass filters. IEEE Trans Microwave Theory Tech. 2013;61(1):69-76.

How to cite this article: Qu M, Li M, Yao L, Rao M, Li S, Deng L. A novel circularly polarized filtering antenna with high out-of-band radiation rejection level. Microw Opt Technol Lett. 2017;60:134-140. https:// doi.org/10.1002/mop.30929
Received: 14 June 2017

DOI: $10.1002 /$ mop.30933

Metamaterial-inspired seriesfed frequency reconfigurable array with zero-phase CRLH interconnects

\author{
Bilal Ijaz' | M. S. Khan' \\ S. M. Asif ${ }^{3}$ | Dimitri E. Anagnostou ${ }^{4}$ \\ Benjamin D. Braaten ${ }^{3}$ \\ ${ }^{1}$ Department of Electrical Engineeinrg, COMSATS Institute of Information \\ Technology, Islamabad, Pakistan \\ ${ }^{2}$ Dipartimento di Ingegneria dell'Informazione, University of Padova, \\ Padova 35131, Italy \\ ${ }^{3}$ Department of Electrical and Computer Engineering, North Dakota State \\ University, Fargo, North Dakota 58102 \\ ${ }^{4}$ Department of Electrical and Computer Engineering, South Dakota School \\ of Mines and Technology, Rapid City, South Dakota 57701
}

Correspondence

M. S. Khan, Dipartimento di Ingegneria dell'Informazione, University of Padova, Padova, 35131, Italy.

Email: mskj786@hotmail.com

\begin{abstract}
Devices with multiband wireless system capabilities have significantly benefited from the introduction of frequency reconfigurable antennas. However, much of the development on frequency reconfigurable antennas has been on single antenna elements. In this letter, a frequency reconfigurable series-fed array is presented that has the benefits of broad-side radiation at two different frequencies. The proposed antenna consists of four reconfigurable dipoles interconnected with reconfigurable zero-phase composite right-/left-handed (CRLH) transmission lines (TLs). PIN diodes are then used to switch between the two measured frequency bands of $2.04-2.18 \mathrm{GHz}$ and $2.44-2.58 \mathrm{GHz}$. The developed prototype presented here showed a near broadside radiation pattern in both switching bands and a maximum gain of $2.1 \mathrm{dBi}$ was measured in both switching bands. It is shown that instead of meander-line interconnects between the dipole elements, much more compact frequency reconfigurable CRLH-TLs can be used.
\end{abstract}

\section{KE Y W O R D S}

composite right-/left-handed transmission lines, frequency reconfigurable antennas, PIN diodes 